\title{
Tribomechanical Properties of the Modified Surface of Alloys of the WC-Co System
}

\author{
Tatyana Oskolkova ${ }^{1}$, Sergey Yaresko $^{2, *}$, and Sergey Balakirov ${ }^{2}$ \\ ${ }^{1}$ Siberian State Industrial University, 654007 Novokuznetsk, Russia \\ ${ }^{2}$ Samara Branch of P.N. Lebedev Physical Institute, 443011 Samara, Russia
}

\begin{abstract}
The research results of tribological characteristics of the surface hardened by electric explosion alloying of tungsten-cobalt hard alloys with carbon, titanium and aluminum are presented. The correlation of tribomechanical properties of the modified surface of alloys with changes in the structure and phase composition of the surface layer of the material is established. It is shown that the best wear characteristics are achieved during hardening of alloys by electric explosion of titanium. In this case, the coefficient of friction decreases by almost 3 times, the wear resistance estimated by the depth of the wear track increases by more than 10.0 times, and by the area of the wear track - by 53.2 times.
\end{abstract}

\section{Introduction}

The increase of durability and reliability of hard alloys can be achieved not only by technological methods in the process of their production, but also by changing the properties of structural components of already sintered hard alloy products [1-3]. Processing of the materials surface with concentrated energy flows (laser, plasma, electron, ion implantation, etc.) in order to improve the level of material properties and create alloys with a new set of physical and mechanical properties is a promising way to modify the characteristics of machine parts and tools. Due to its features, it can be used to effectively control the structure formation in the surface layer of the material most susceptible to wear. The field of application of surface hardening methods is increasingly expanding, and now they are used not only in scientific laboratories, but also in industrial applications.

Use of external energy impact to increase the level of material properties and create alloys with new physical and mechanical properties is intended for purposeful control of performance characteristics of hard alloy products. A reasonable choice of the most effective methods and specific modes of strengthening treatment of a material is impossible without the accumulation of experimental and theoretical data on changes in the structure in the irradiated layers and its influence on its properties.

This paper considers one of the promising methods of surface modification electroexplosive alloying (EEA) of tungsten-cobalt hard alloys [4]. The EEA method involves heating the surface and saturating it with explosion products, followed by selfhardening by removing heat deep into the material and the environment. The instrument of

\footnotetext{
*Corresponding author: yarsi54@gmail.com
} 
thermal action on the surface and the source of alloying elements during EEA is a pulsed multiphase plasma jet formed from the material of the exploded conductor.

The aim of this work was to experimentally evaluate the tribological properties of the surface of WC-Co hard alloys modified using EEA and their relations to structural and phase changes in the surface layer of the alloys after processing.

\section{Equipment and research methods}

In this work, the study object was a hard alloy VK10KS (Co - up to $10 \%$, WC grain size 5-8 $\mu \mathrm{m}$ ), pre-hardened by electrophysical methods. Single-component EEA of the alloy surface with carbon, aluminum, and titanium was considered as a hardening method.

The surface treatment of the hard alloy VK10KS by the EEA method was carried out at an exposure intensity of $6.0 \mathrm{GW} / \mathrm{m}^{2}$, which provided surface melting and convective mixing of the melt when exposed to a plasma jet formed from the alloying element material on the alloy surface.

The structure features of the hard alloy surface layer after its modification, the content of alloying elements and the depth of the plasma exposure zone were determined using a Philips SEM 515 scanning electron microscope equipped with an EDAX Genesis microanalyzer. X-ray phase studies were carried out using a DRON-2.0 diffractometer in $\mathrm{Fe}_{K \alpha}$-radiation.

Tribological tests of the samples in the initial state and after treatment were carried out using a "PC-Operated High Temperature Tribometer" at room temperature. The wear of the samples was determined using a high-precision measuring device "Micro Measure 3D station" with software by measuring the depth and cross-sectional area of the wear track after testing. The track was formed as a result of the action of a stationary diamond indenter on a rotating sample at a load of $3 \mathrm{~N}$ and a number of revolutions of 4000 . Nanoindentation of the surface of the hard alloy was carried out on the device "Nano Hardness Tester" of the company CSEM.

\section{Results and discussion}

\subsection{Studies of the structure and phase composition of the alloy after EEA}

According to the results of electron microscopic, X-ray phase and durametric studies of the modified VK10KS alloy, it was found that with a single-component EEA with all exploding conductors in the surface layer of the alloy with a thickness of 15-40 $\mu \mathrm{m}$, new high-solid phases consisting of elements of the base material and exploding conductors (TiC, $\mathrm{W}_{2} \mathrm{C}, \mathrm{Al}_{2} \mathrm{O}_{3}$ ) are formed during crystallization.

The most alloying depth $(\sim 40 \mu \mathrm{m})$ with increased performance characteristics (Table 1) was achieved when the alloy was treated with titanium electroexplosion products. In this case, a three-layer alloying zone is formed (Figure 1): the surface (top) layer is enriched with carbides $(T i, W) C-64 \%, T i C-16 \%, W C-11 \%$ и $W_{2} C-9 \%$ with high hardness; the intermediate layer consists mainly of tungsten semicarbide $W_{2} C$ with a hardness higher than that of tungsten monocarbide $W C$, and the third layer, in which changes are recorded only in the Co-binder, associated with its additional alloying with both explosion products and the base material, and with the formation of a deformation slip bands (Figure 2) and dislocations. The content of titanium in the Co-binder is $0.04 \%$, of tungsten $-20.02 \%$. Then there is a smooth transition to the base material without cracking. The total depth of the plasma exposure zone is $30-40 \mu \mathrm{m}$. 
Table 1. Properties of the hard alloy VK10KS after a single-component EEA.

\begin{tabular}{|l|c|c|c|c|}
\hline $\begin{array}{c}\text { Coating material } \\
\text { (exploding conductor) }\end{array}$ & $\begin{array}{c}\text { Nano-hardness, } \\
\mathrm{MPa}\end{array}$ & $\begin{array}{c}\text { Wear track } \\
\text { depth, } \mu \mathrm{m}\end{array}$ & $\begin{array}{c}\text { Wear track } \\
\text { area, } \mu \mathrm{m}^{2}\end{array}$ & $\begin{array}{c}\text { Friction } \\
\text { coefficient, } \mu\end{array}$ \\
\hline initial state & 12,000 & 58.0 & 12,921 & 0.41 \\
\hline carbon & 20,000 & 11.7 & 1,495 & 0.23 \\
\hline aluminum & 23,500 & 9.02 & 948 & 0.22 \\
\hline titanium & 25,000 & 5.40 & 243 & 0.14 \\
\hline
\end{tabular}

In the case of EEA of a hard alloy with carbon, a decrease in the size of tungsten carbide grains is observed during crystallization after melting during processing with a power density of $6.0 \mathrm{GW} / \mathrm{m}^{2}$. Electron microscopy data confirm the increase in the degree of dispersion of the carbide phase after treatment. The depth of the modified near-surface layer, associated with a decrease in the grain size of tungsten carbides, is 10-15 $\mu \mathrm{m}$.

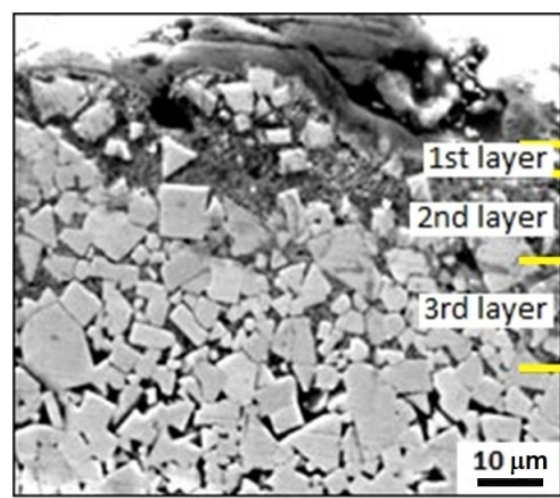

Fig. 1. Microstructure of the alloy after electroexplosion treatment of titanium.

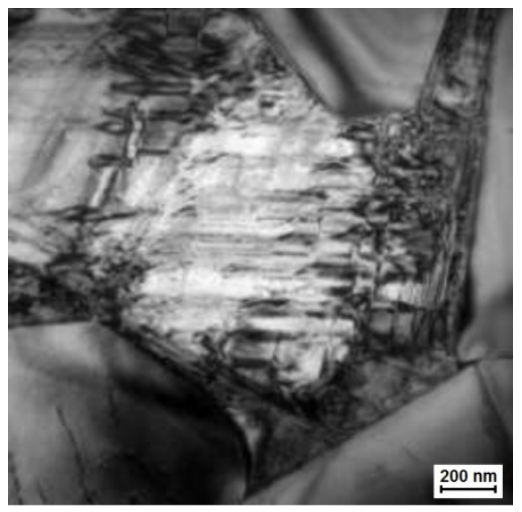

Fig. 2. Structure of the alloy after electroexplosion treatment with titanium at a distance of $20 \mu \mathrm{m}$ from the surface.

In addition to reducing the grain size of tungsten carbides, a change in the chemical composition of the Co-binder in the layer located at a distance of $15-20 \mu \mathrm{m}$ from the surface was recorded by scanning electron microscopy. Figure 3 (b) clearly shows the changes in the composition of the Co-phase after EEA in contrast to the initial state (Figure 3 (a)). At EEA with carbon-graphite fibers, the content of tungsten in the Co-binder increases to $32.96 \%$ (in the initial state $-10.89 \%$ ) and is accompanied by the release of fine tungsten carbides $W C$ and $W_{2} C$ from a supersaturated cobalt solid solution.

The formation of a solid solution more alloyed with tungsten and the resulting decrease in the difference between the coefficients of thermal expansion of the $W C$ and the Co-phase cause a decrease in the stress state in the Co-phase, which has a positive effect on the operational stability of the hard alloy as a whole.

According to X-ray phase analysis, after EEA with carbon instead of tungsten monocarbide $W C$, a higher-hardness tungsten semicarbide $W_{2} C(60 \%)$, a small amount of unstable carbide $W_{l-x} C(17 \%)$ and $W C(23 \%)$ are formed in the surface layer.

When processing the VK10KS alloy by electric explosion of aluminum foil, a layer with a chemical composition of $84.11 \% \mathrm{~W} ; 6.89 \% \mathrm{Al} ; 6.11 \% \mathrm{O}_{2} ; 2.89 \% \mathrm{C}$ and a thickness of $20-25 \mu \mathrm{m}$ is formed on the surface of the alloy, smoothly passing into the base. No microcracks were found at the boundary of the alloying zone and the base material. The size of the tungsten carbides in the surface zone is reduced to $1.0-1.5 \mu \mathrm{m}$. According to the XRD data, a small amount of tungsten monocarbide (7\%), $\alpha-\mathrm{Al}_{2} \mathrm{O}_{3}$ oxide $(13 \%)$, and 
tungsten semicarbide $\mathrm{W}_{2} \mathrm{C}(80 \%)$ is formed on the alloy surface after EEA with aluminum.

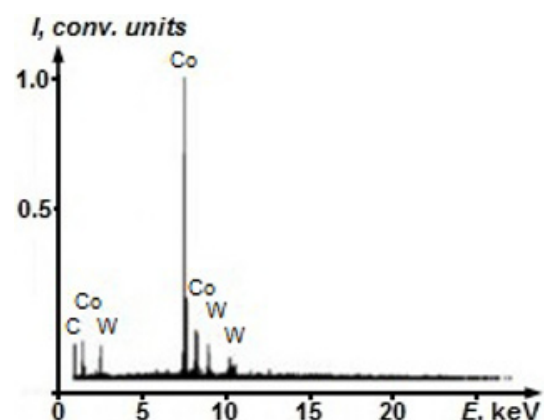

a - initial state

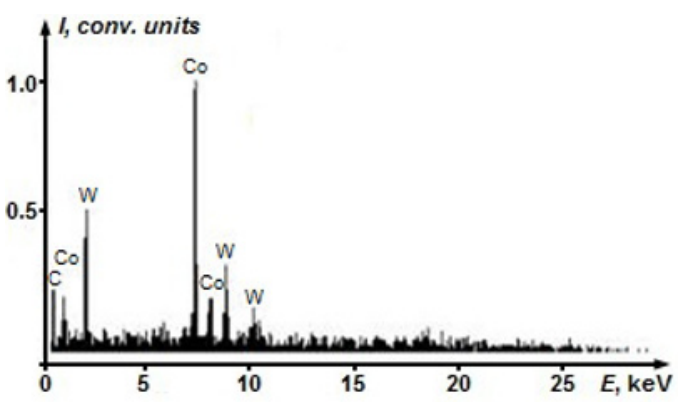

$\mathrm{b}$ - after processing

Fig. 3. Distribution of elements in the Co-binder of the VK10KS alloy after treatment with an electroexplosion of carbon.

In addition, the cobalt bond is alloyed with aluminum. $0.14 \%$ of aluminum was dissolved in the cobalt bond of the hardened layer (Figure 4). According to [5], additional alloying of the cubic modification of cobalt $(\beta-\mathrm{Co})$ leads to its stabilization and allows us to explain the increase in the hardness, wear resistance and bending strength of the alloy.

A more detailed study of the structure of the VK10KS alloy after EEA with aluminum, as with EEA with titanium, revealed the presence of deformation bands on the carbide grains (Figure 5).

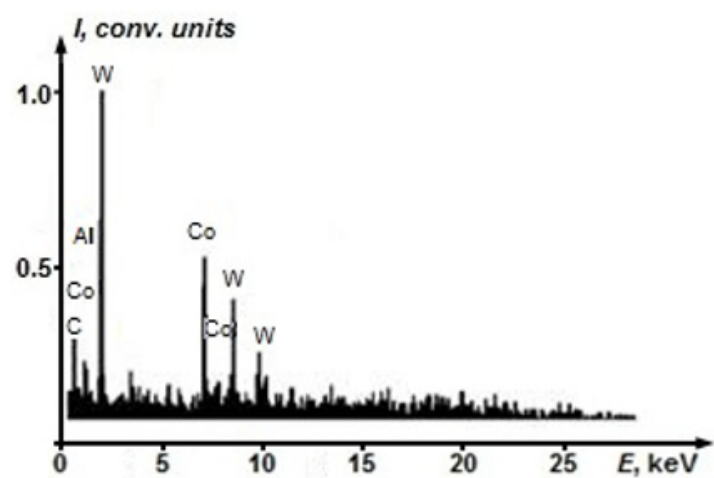

Fig. 4. The elemental composition of the Co-binder in the surface layer of the VK10KS alloy after alloying by electroexplosion of aluminum.

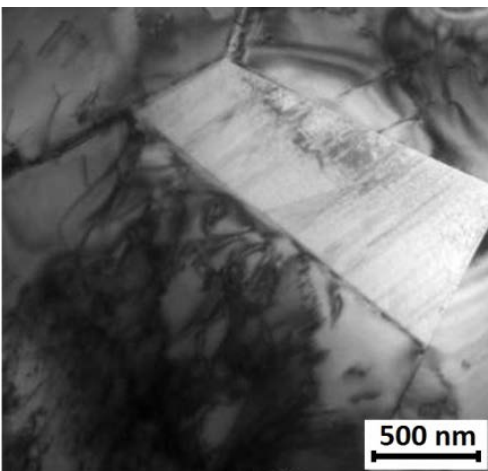

Fig. 5. Deformation bands on carbide grains after EEA with aluminum at a distance of $20 \mu \mathrm{m}$ from the surface.

Studies of the composition of the Co-binder in the heat-affected zone after EEA showed that for all exploded conductors, the Co-binder is additionally alloyed with chemical elements that are part of the exploded and basic materials, namely, tungsten (25.60 $32.96 \%)$, carbon, aluminum ( $0.14 \%$ in the explosion of an aluminum foil), titanium $(0.2 \%$ in the explosion of a titanium foil). In addition, it was found that during EEA, finely dispersed tungsten carbides are formed in a supersaturated solid solution of cobalt. An increase in the degree of alloying of the cobalt binder $(\beta-\mathrm{Co})$ leads to its stabilization, an increase in the softening temperature, which will positively affect the operational stability of the alloy. 


\subsection{Tribological studies}

The study of the modified EEA of the hard alloy VK10KS showed that the structures formed in the surface layer have a complex of physical and mechanical characteristics that significantly exceed the characteristics of the source material. For all types of singlecomponent EEA, the hard alloy after processing has a high hardness and increased wear resistance compared to the initial state. These properties are provided by the structures formed in the surface layer. In EEA with carbon is the carbides $W_{2} C, W_{1-x} C$, and $W C$. The newly formed phases are located along the boundaries separating the crystals of the initial tungsten carbide and the Co-bond, and have sizes from 30 to $80 \mathrm{~nm}$. This allows us to talk about the nanostructuring of the surface, which leads to its hardening. When treated with aluminum, mainly the $\mathrm{W}_{2} \mathrm{C}$ and $\alpha-\mathrm{Al}_{2} \mathrm{O}_{3}$ phases, as well as tungsten monocarbide, are formed on the surface of the treated alloy. When treated with titanium, the performance characteristics of the alloy are determined by the top layer consisting of $\mathrm{TiC}$ and $(\mathrm{Ti}, \mathrm{W}) \mathrm{C}$.

The presence of these structures, as well as a sufficiently developed dislocation structure and the established increase in the degree of Co-phase alloying, have a favorable effect on the operational stability of WC-Co alloys and determine the high tribological properties of the modified surface. The results of studies of the wear and mechanical characteristics of the VK10KS alloy after single-component EEA with various elements are presented in Table 1.

The wear of the samples was determined by measuring the depth and area of the wear track before and after hardening. A typical pattern of the wear track is shown in Figures 6 and 7. A similar behavior of the alloy after EEA is observed for all the cases of hardening treatment under consideration, for example, the characteristic wear tracks for EEA with carbon and aluminum are shown in Figures 8 and 9. When single-component alloying of the surface of the VK10KS alloy with a pulsed plasma jet, the greatest effect is achieved when titanium explodes. At the same time, a maximum processing depth of about $40 \mu \mathrm{m}$, a surface nanohardness of $25,000 \mathrm{MPa}$, a low coefficient of friction $\mu=0.14$ (Figure 10), a wear track depth of $5.4 \mu \mathrm{m}$ (Table 1, Figure 7), and in the initial state $\mu=0.41$ and a wear track depth of $58.0 \mu \mathrm{m}$ (Figure 6) are achieved. Such low wear rates are provided by the structural state of the alloy surface, which is based on titanium and tungsten carbides.

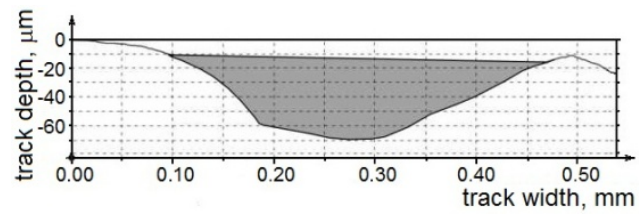

Fig. 6. Profile and area of the wear track of the original sample.

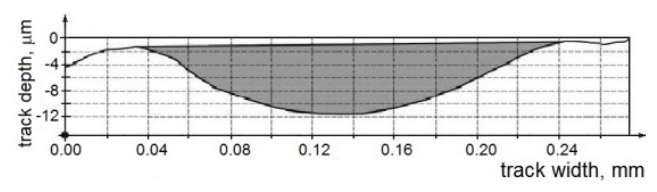

Fig. 8. Profile and area of the wear track of the sample after EEA with carbon.

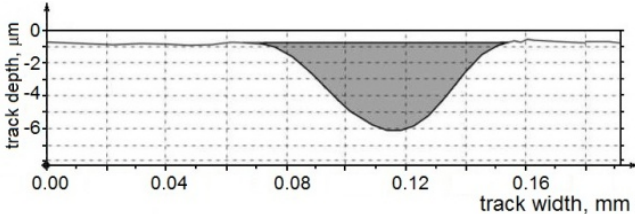

Fig. 7. Profile and area of the wear track of the sample after EEA with titanium.

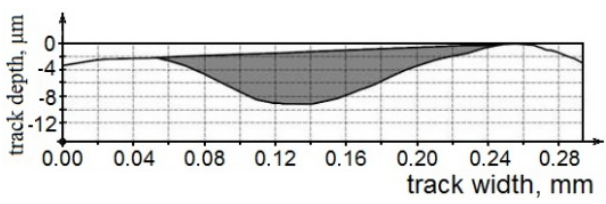

Fig. 9. Profile and area of the wear track of the sample after EEA with aluminum.

The change in the composition of the hard alloy surface after EEA with carbon could 
not but affect the change in the mechanical properties of the modified layer. The test results showed that the depth of the wear track after processing is $11.7 \mu \mathrm{m}$ (Figure 8), and the cross-sectional area of the track of worn samples is $1,495 \mu \mathrm{m}^{2}$ (Table 1). The decrease in the wear of samples after processing is associated with a change in the type of tungsten carbide in the surface layer and its grinding. For this type of surface treatment, the value of the friction coefficient after running-in is set to 0.23 .

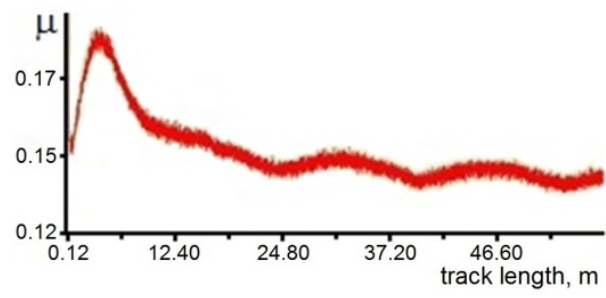

Fig. 10. Coefficient of friction of the alloy after EEA with titanium.

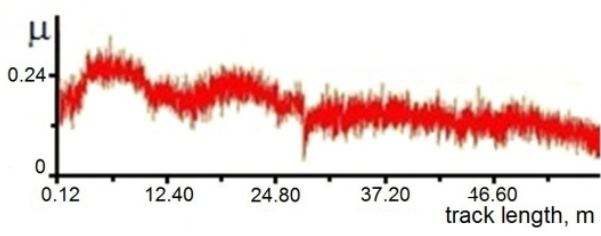

Fig. 11. Coefficient of friction of the alloy after EEA with aluminum foil.

Nanoindentation showed that for the alloy after EEA with aluminum, the hardness increases to the values of $23,500 \mathrm{MPa}$. At the same time, the obtained values exceed the nanohardness of the samples in the initial state by 1.5-2 times. The recorded increase (Table 1, Figure 9) in the wear resistance of the VK10KS hard alloy after electroexplosion treatment of aluminum foil is associated with the formation of fine new phases on the surface $-\mathrm{W}_{2} \mathrm{C}$ and $\mathrm{Al}_{2} \mathrm{O}_{3}$ with high hardness (Table 1) [6]. The depth and area of the wear track are $9.02 \mu \mathrm{m}$ and $978 \mu \mathrm{m}^{2}$, respectively (Figure 9, Table 1). The value of the coefficient of friction in the steady state is almost 1.9 times less than for the alloy in the initial state (Table 1, Figure 10-11).

As already noted, EEA for all types of exploding conductors leads to additional alloying of the Co-phase with tungsten, carbon, aluminum, and titanium, which are part of the exploding materials and the base. The established increase in the degree of doping of the Co-phase stabilizes the FCC lattice $(\beta-\mathrm{Co})$, which positively affects the operational resistance of WC-Co hard alloys during cutting.

It is known that at high cutting speeds, high temperature and large plastic deformation in the contact zone contribute to the diffusion dissolution of the components of the tool and processed materials. As a result of the diffusion of $\mathrm{Fe}$ by the substitution mechanism, the binder is a solid solution of $\mathrm{W}, \mathrm{C}$, and $\mathrm{Fe}$ in $\mathrm{Co} . \alpha$-WC carbide can react with Fe dissolved in Co to form double carbides of the $\mathrm{Fe}_{3} \mathrm{WC}$ type and free carbon [7]. These changes in the structure at the cutting edge cause increased tool wear at high cutting speeds [8]. The decrease in the strength of the carbide tool in this case is due to the greater ability of iron to dissolve tungsten carbide in the solid state. In this case, the binding Co-phase loses its plasticity, and the entire alloy becomes harder and more brittle.

After the hardening treatment, the presence of excess $\mathrm{W}$ and alloying elements dissolved in Co complicates the diffusion of $\mathrm{Fe}$ into $\mathrm{Co}$ and the formation of iron-tungsten carbides during the cutting process, leads to an increase in the resistance to the destruction of the binding phase, which is manifested in a decrease in the embrittlement of the surface layers of the alloy during the operation of the tool. There is an increase in the uniformity of the structure and properties of the surface layer of the alloy after hardening, when cutting, this is manifested in a decrease in the coefficient of variation of tool life. According to [9], in this case, the stability of the cutting process is increased at high flow rates under conditions of diffusion wear of the carbide tool. 


\section{Conclusions}

1. It is experimentally established that the treatment of a hard alloy VK10KS by a pulsed multiphase plasma jet formed from the material of the exploding conductor at a power density of $6.0 \mathrm{GW} / \mathrm{m}^{2}$ leads to the creation of a hardened layer up to $40 \mu \mathrm{m}$ thick, consisting of finely dispersed high-hard carbides and/or oxides. The composition, structure and properties of the surface layer of the modified alloy are determined by the material of the exploding conductor.

2. It is established that the EEA of hard alloys leads to a significant change in the tribological characteristics of its surface. The nanohardness of the surface increases by 1.67 ... 2.08 times and reaches values of $20,000 \ldots 25,000 \mathrm{MPa}$. The wear resistance, estimated by the depth of the wear track, increases from 4.9 to 10.7 times, and by its area - from 8.6 to 53.2 times, the coefficient of friction decreases to values of $0.14 \ldots 0.23$ against the value of 0.41 for the untreated surface.

3. The experimentally recorded additional alloying of the Co-phase with tungsten, carbon, and alloying elements has a positive effect on the operational resistance of WC-Co hard alloys during cutting. Under the conditions of diffusion wear of the carbide tool, the effect is to increase the stability of the cutting process at high turning speeds.

\section{References}

1. S. Yaresko, Physical and Technological Bases of Hardening of Hard Alloys (Samara, Publishing house: Samara Scientific Center, Russian Academy of Sciences, 2006) (in Russian)

2. T. Oskolkova, A. Glezer, Steel Transl., 47, 788-96 (2007)

3. A. Lisovskii, J. Superhard Mater., 32, 250-62 (2010)

4. A. Bagautdinov, E. Budovskih, Yu. Ivanov, V. Gromov, Physical Bases of Electroexplosive Alloying of Metals and Alloys (Novokuznetsk, SibGIU, 2007) (in Russian)

5. V. Panov, A. CHuvilin, Technology and Properties of Sintered Hard Alloys and Their Products (Moscow, MISIS,2001) (in Russian)

6. G. Samsonov, I. Vinickij, Refractory Compositions (Moscow, Metallurgiya, 1976) (in Russian)

7. O. Bannyh et al., Diagrams of the State of Dual and Multicomponent Systems Based on Iron (Moscow, Metallurgiya, 1986) (in Russian)

8. T. Loladze, Strength and Wear Resistance of the Cutting Tool (Moscow, Mashinostroenie, 1982) (in Russian)

9. S. Yaresko, Russ. J. Non-Ferrous Metals, 50, 556-62 (2009) 